\title{
CME Nuclear medicine (108462): self-assessment questionnaire
}

\author{
Edited by Sabina Dizdarevic and Tahseen A Chowdhury
}

SAQs and answers are ONLINE for RCP fellows and collegiate members

SAQs and answers are ONLINE for RCP fellows and collegiate members

\section{Format}

Candidates are asked to choose the best answer from the five possible answers. This best of five format is used in many medical examinations, however the questions are not intended to be representative of those used in the MRCP(UK) Part 1 or Part 2 Written Examinations.

\section{The answering process}

1 Go to www.rcplondon.ac.uk/SAQ

2 Log on using your usual RCP username and password

3 Select the relevant CME question paper

4 Answer all 10 questions by selecting the best answer from the options provided

5 Once you have answered all the questions, click on Submit

\section{Registering your external CPD credits}

Carrying out this activity allows you to claim two external CPD credits. These will be automatically transferred to your CPD diary, where you can review the activity and claim your points.

1. Positron emission tomography/computerised tomography

(a) can be used for detecting the source of sepsis in problematic cases and in pyrexia of unknown origin

(b) imaging with amyloid tracers is specific for Alzheimer's disease

(c) is not currently funded for non-oncological indications in the UK

(d) should be reserved only for the assessment of high-risk solitary pulmonary nodules

(e) uses fludeoxyglucose as a universal imaging agent for all types of cancer.

2. Contrast agents for use in combined modality imaging

(a) can provide images at both whole body and cellular level if they incorporate optical contrast

(b) cannot be used in conjunction with single-photon emission computerised tomography imaging

(c) confer the advantage of lower absorbed radiation dose compared with conventional imaging
(d) must contain a radionuclide for use during surgery
(e) must contain more than one type of contrast in a single entity to be useful.

3. Which radiopharmaceutical has demonstrated survival benefit in patients with bone metastases from prostate cancer?
(a) Iodine-131
(b) Radium-223
(c) Strontium-89
(d) Samarium-153
(e) Technetium-99m.

4. Which of these is the most common organ for metastases from castration-resistant prostate cancer?
(a) bone
(b) brain
(c) liver
(d) lung
(e) spleen.

5. Which of the following is true regarding peptide receptor radionuclide therapy (PRRT)?
(a) The most commonly used PRRT agent is ${ }^{90} \mathrm{Y}$-DOTATATE.
(b) PRRT is indicated as first-line therapy for patients with grade 2 neuroendocrine tumours.
(c) Renal impairment is more likely to occur following ${ }^{90}$ Y-DOTATATE rather than ${ }^{177}$ Lu-DOTATATE.
(d) Renal toxicity is the most common side effect.
(e) Somatostatin receptor uptake scan is not required for selection for PRRT.

6. Which of the following is true regarding metaiodobenzylguanidine (MIBG)?
(a) ${ }^{123}$ I-MIBG is the functional imaging agent of choice in the majority of neuroendocrine tumours.
(b) MIBG is a catecholamine analogue.
(c) MIBG is taken up and stored in the cytoplasm of the tumour cells.
(d) MIBG therapy is performed using ${ }^{123}$ I-MIBG.
(e) The target is the somatostatin receptor.

7. ${ }^{131}$ I-radioiodine is used in the treatment of benign and malignant thyroid disease because of its
(a) alpha-particle emission
(b) beta- and alpha-particle emission
(c) beta-particle emission 

(d) gamma ray emission
(e) positron emission.

8. In benign thyroid disease, ${ }^{131}$ I-radioiodine is indicated in the treatment of
(a) De Quervains thyroiditis
(b) differentiated thyroid cancer
(c) Graves' disease
(d) Hashimoto's thyrotoxicosis
(e) hyperthyroidism due to any cause.

9. An advantage of yttrium-90 as a radionuclide for selective internal radiation therapy is that
(a) it has a long physical half-life
(b) it emits easy to image gamma rays
(c) it emits short-range beta radiation, resulting in cell damage
(d) it is excreted via the faeces
(e) it is cheap.

10. The first angiogram for selective internal radiation therapy is required to
(a) identify the liver arterial anatomy
(b) embolise the gastroduodenal artery, if necessary
(c) administer technetium-99m macroaggregated albumin and determine the lung shunt fraction
(d) all of the above
(e) none of the above.

\section{CME Geriatric medicine SAQ}

Answers to the CME SAQ published in Clinical Medicine in August 2017
Q1 Q2
Q3
Q4 Q5
Q6 Q7
Q7 Q8
Q9 Q10
(c)
(e) (d)
(d) (c)
(a) (d)
(d) (d)
(c) 